\title{
LOW-TEMPERATURE ATMOSPHERIC-PRESSURE PLASMA IN MICROBIAL DECONTAMINATION AND MEAT TECHNOLOGY.
}

\section{A REVIEW}

\author{
Natalia A. Gorbunova
}

V.M. Gorbatov Federal Research Center for Food Systems of Russian Academy of Sciences, Moscow, Russia

Keywords: low-temperature plasma, plasma-based inactivation, microorganisms, meat products, nitrite

\begin{abstract}
The development of plasma technology is associated with the unique features of non-equilibrium low-temperature plasma: high electron energy and high concentration of chemically active excited and charged particles at low gas temperature, which allows to process thermolabile materials and biological objects in gentle conditions when high temperatures are not required. The biological effects of low-temperature plasma exposure are considered. It was established that during plasma treatment, a combined effect on cells and tissues of living systems from UV radiation, ions and chemically active particles occurs. Depending on the plasma type, the significance of each of the listed mechanisms for increasing the effectiveness of plasma treatment may vary. However, all these mechanisms interact with each other and have a synergistic effect. It was shown that the conducted studies confirm the ability of low-temperature plasma to inactivate pathogenic microorganisms upon contact with biological objects and foods. The results of the studies are presented, the purpose of which was to study the effect of plasma treatment on nitrite concentration in the water treated by this method and to assess the possibility of its use as a source of nitrite when curing meat products.
\end{abstract}

\section{Introduction}

In recent years, cold plasma technology has been used in chemical technology, metal processing and production of various coatings, medicine, and food industry, in particular, as a low-temperature sterilization method that provides a high level of microbial inactivation for water purification and disinfection of premises. The potential size of the cold plasma equipment market in Russia and the EU amounts to $\$ 22$ billion with a growth potential of 7 to $8 \%$ per year [1]. To implement these technologies in industry, various plasma installations have been developed, in which thermal and plasma-chemical treatment of substances is realized.

The technology for cold plasma production with the temperature of the torch visible part of 40 to $42{ }^{\circ} \mathrm{C}$ is known for a long time. The main difference between cold plasma and other plasms types is that the temperature of the former is close to the temperature of biological objects. This fact allows applying this type of plasma to living systems [2].

\section{The concept of plasma}

Plasma is often considered the fourth physical state of matter. The English chemist and physicist W. Crookes introduced this concept in 1879 to describe the ionized medium of a gas discharge. When solid substance is heated, it passes into a new state, usually liquid. In turn, the liquid passes into a gas when heated. Its further heating leads to the ionization of atoms. Gas in which a significant part of atoms or molecules is ionized is called plasma [3].

Plasma is a complex, quasi-neutral multicomponent system including plasma radiation, both positively and negatively charged particles, and electroneutral particles, which, however, are chemically active (radicals, excited atoms and molecules). Thus, plasma acts simultaneously as a source of radiation, part of which has bactericidal properties, and as a chemically active medium $[4,5]$.

Electrolyte solutions, semiconductors and electricdischarge plasma generated in the range of low and atmospheric pressure of $10-2$ to $103 \mathrm{~Pa}$ have similar properties. At this pressure, the plasma is a partially ionized gas with a degree of ionization less than 10-4, in which the concentration of electrons, positive and negative ions, radicals is sufficient to maintain a quasi-neutral state [6].

Plasma is often classified into low-temperature and high-temperature, as well as into equilibrium and nonequilibrium. Low-temperature plasma, in turn, is divided into thermal and non-thermal plasma. In non-equilibrium plasma, electron temperature significantly exceeds the total temperature of ions. In equilibrium plasma, the total values of both temperatures are approximately equal, while the equilibrium plasma is often hot [7].

Particles produced in high-temperature plasma are in a state of thermal equilibrium, and, as a result, electrons and heavy ions have a high temperature. Low-temperature thermal plasma is characterized by the equality of electron and gas temperature, which is based on elastic collisions between electrons and heavy particles (ions, molecules, 
and atoms). The energy of the electrons is consumed by heavy particles, which leads to their heating. In a thermal plasma, electrons and heavy particles are in the state of local thermodynamic equilibrium.

\section{Some aspects of the low-temperature plasma} physics

In the plasma state of a gas, vibrational-rotational excitation and dissociation of the molecules occur. Particles formed as a result of electron impact are highly reactive and interact not only with each other, but also with any object introduced into plasma. The plasma state of a substance, even with a low degree of ionization, gives a wide range of chemically active particles, the source of which is not thermal energy used in classical technological processes, but the energy of an external electric field. This causes the so-called non-equilibrium of the electricdischarge plasma due to the low efficiency of energy transfer from the electron to other particles by collisions. As a result, a difference occurs between the translational energy of electrons and more massive particles (ions, radicals). Recalculation of the average electron energy into the corresponding thermal units gives the electron "temperature" of about 3.104 to 1.105 K [8].

Low-temperature plasma is considered equilibrium if its components are in thermodynamic equilibrium, i.e. the temperatures of electrons, ions and neutral particles are equal. In a low-temperature plasma, non-equilibrium conditions are easily created as a result of the selective action of external electric fields: the electrical energy from them is transferred to charged particles, and then to gas particles by collisions. With this method of energy introducing, the average energy of charged particles may significantly differ from the thermal energy of neutral particles. First, this refers to electrons, which, due to their small mass, inefficiently exchange energy during elastic collisions with neutral gas particles. In this case, not only the average electron energy, but also the form of energy distribution of electrons may significantly differ from the equilibrium [7].

For non-thermal plasma, it is characteristic that the temperature of electrons is significantly higher compared to the temperature of ions and neutral gas. This temperature difference depends on the frequency of collisions between electrons and heavy particles. Due to the small loss of kinetic energy in elastic collisions between electrons and heavy particles, the electron energy remains high. Therefore, non-thermal plasma is not in a state of local thermodynamic equilibrium. Because the temperature of neutral gas is equal to or close to room temperature, nonthermal plasma is often called a "cold" plasma [9].

In a non-thermal plasma, free electrons are very "hot" and have temperatures of several thousand degrees Kelvin $(\mathrm{K})$, while neutral particles and ions remain "cold". Due to high-energy collisions, excited electrons form a whole spectrum of radicals and excited particles with high reactivity. This combination of low temperatures with high reactivity makes non-thermal plasma a technologically advantageous and very effective tool for performing technological processes that would require the use of very high temperatures and harmful corrosive chemicals even if they were feasible without plasma [9].

An important feature of non-equilibrium cold plasma is its ability to generate a unique 'one pot' cocktail of biologically active agents, such as reactive oxygen species and reactive nitrogen species, while remaining close to ambient temperature, which enables its safe application to biological materials including foods. Reactive particles and their concentration in plasma will vary depending on many factors including the gas, in which the plasma is induced, the configuration of plasma source, the consumed power of gas, the duration of treatment and the moisture level in the product [10].

Depending on the mechanism for obtaining lowtemperature plasma, the following types of discharges are distinguished: a glow discharge, a dielectric barrier discharge, a corona discharge, and plasma jets. Non-toxic gases such as argon, helium, nitrogen, oxygen, air, water vapor, and their combinations are most commonly used as gas mixtures.

\section{Biological effects of exposure to non-equilibrium low-temperature plasma}

It has been established that during plasma treatment, a combined effect on cells and tissues of UV radiation, ions and chemically active particles is observed [11,12]. Depending on the type of plasma, the significance of each of these mechanisms for sterilization efficiency may vary. However, all these mechanisms interact with each other and have a synergistic effect $[13,14]$.

Ions and chemically active particles interact with the outer layers of spores and bacteria, causing its erosion and loss of integrity. At the same time, chemically active particles and ions of the plasma-forming gas are adsorbed on the surface of bacteria and chemically react with the cell membrane molecules forming toxic compounds and secondary radicals. Such damage leads to the release of individual microorganisms from the matrix (dirt, biofilm, bacterial clump) on the surface of sterilized object. By removing the outer layers, plasma-induced erosion reduces shielding from UV radiation, which can cause direct destruction of the microbial genetic material.

Pulsed electrical discharges cause the formation of defects in the cell membrane of bacteria, which allows highly reactive oxidizing agents to freely penetrate into the internal environment of cells and disrupt their metabolic processes. This principle is used to inactivate microorganisms. Cells die as a result of ruptures in cell membrane that protects bacteria from the external environment $[15,16]$.

The most significant biological effect of plasma on bacterial cells and living tissues is caused by the reactive 
oxygen species (ozone, atomic oxygen, superoxide anion radical, peroxides, hydroxyl radicals) and reactive nitrogen species (for example, NO). In atmospheric-pressure plasma, reactive neutral particles (atomic oxygen, singlet oxygen, ozone) are mainly generated, and in low-pressure plasma, ions are predominantly produced.

The interaction of plasma electrons with cell membrane violates its structure. The accumulation of charged particles on the outer bacterial membrane may exceed its tensile strength and, thus, cause its damage. This mechanism is most likely in plasma treatment of gramnegative bacteria; their membrane is thinner than that of gram-positive microorganisms, and its structure is not so well arranged [17,18].

According to some authors, UV radiation is the dominant mechanism of the bactericidal action of lowpressure plasma $[15,19]$. UV radiation with wavelengths less than $300 \mathrm{~nm}(220-280 \mathrm{~nm})$ penetrates deep into cells and causes breaks in DNA leading to the death of microorganisms and inhibition of their reproduction [11]. In addition to ruptures, UV radiation contributes to the formation of thymine dimers in DNA molecules, which prevents the bacterial cell from replicating the genetic material $[19,20]$.

A study by the Russian scientists [21] showed that the treatment of E. coli cells with cold plasma results in partial or complete loss of integrity of the cell cytoplasmic membrane, which is accompanied by the release of intracellular compounds into the extracellular environment. Quantitative assessment of the cell membrane damage showed that a loss of at least $23.6 \%$ of intracellular compounds is enough for cell death (calculated from the release of intracellular nucleotides). The use of media with different ionic strength to create osmotic shock showed that the treatment of E. coli cells with cold plasma resulted in a significant decrease of their membrane strength. The authors assumed that the cell membrane and cytoplasmic membrane are the first to be exposed to plasma active particles. Only after the active particles have passed through the damaged cell membranes, they may damage the DNA and key cell enzymes. At the same time, it was noted that the study of the mechanism for microbial inactivation by the cold plasma is only at initial stage. A clear understanding of this phenomenon will increase the efficiency of plasma-based sterilization, accelerate the widespread introduction of a new sterilization method and can help in solving current problems in the field of biosafety and in other areas where fast and effective microbial decontamination is required.

4. Use of low-temperature plasma as an antimicrobial agent. Plasma-based sterilization.

An important task is to determine the mechanisms and assess the effect of low-temperature plasma on microorganisms.

The advantages of plasma sterilization methods include the possibility of decontaminating materials that are sensitive to heat (the treatment temperature does not exceed $50{ }^{\circ} \mathrm{C}$ ), the absence of auxiliary chemical compounds formation that are hazardous for the environment and human health $[22,23]$ and a reduction in treatment duration [14].

It should be noted that at the current stage of research, the assessment of plasma-based microbial inactivation results obtained under various experimental conditions is a big problem [9].

For the first time, W. Siemens et al. [24] applied corona discharge for water purification from biological contaminations in 1857. Later, corona discharge efficiency was confirmed both in aqueous suspensions of bacteria [25] and in the sterilization of solid surfaces contaminated by microorganisms and their spores [26]. It has been established that for complete elimination of various grampositive and gram-negative bacteria in corona discharge, 5 to $15 \mathrm{~min}$ exposure is necessary depending on the discharge power $[8,13,14,21,27]$. At the same time, the efficiency of plasma-based sterilization may be $99.9 \%$.

The efficiency and rate of microbial inactivation depends on the material, on which they are located. It was revealed that the most quickly inactivation of Escherichia coli K12 occurred on a polypropylene surface followed by glass and agar, respectively. An increase in the sensitivity of microorganisms with a decrease in $\mathrm{pH}$ and ambient temperature was noted [28]. Moreover, gram-negative microorganisms are more susceptible to plasma than gram-positive ones, which may be due to the different structure of the cell membrane [29].

Glow discharge has a bactericidal effect not only on single bacteria, but also on biofilms, which are extremely resistant to traditional sterilization methods. Thus, glow discharge destroyed the biofilms of Rhizobium gallicum and Chromobacterium violaceum, which were formed for four and seven days, causing the death of $100 \%$ microorganisms [30,31]. It is especially important that glow discharge may be used when the treated surface is sensitive to external influences (for example, when treating food products), which prevents changes in color and structure and the formation of toxic products [31].

In Tomsk State University [5], the effect of atmosphericpressure plasma treatment of surfaces contaminated with E. coli was investigated. A high-frequency discharge in air at atmospheric pressure was used as a plasma source. The generator created high-voltage pulses with a frequency of $122 \mathrm{kHz}$. The plasma was ignited 0.7 and $2.7 \mathrm{~cm}$ from the substrate, on which a layer of bacteria with a known concentration was applied. After irradiation, the substrate was placed in thermostat for 2 days. On the third day, microorganism colonies were counted to determine the effectiveness of sterilization.

The results of the experiments allow to conclude that the key factors of atmospheric-pressure plasma sterilizing effect under the above conditions on bacterial cultures 
are ultraviolet radiation with $200<\lambda<220 \mathrm{~nm}$ and electroneutral chemically active particles. Effectiveness of this UV radiation band is due to the fact that the maximum inactivation of microbial DNA occurs in this frequency range. The author notes that the latter is particularly interesting, since the plasma radiation intensity in the band of $200<\lambda<220 \mathrm{~nm}$ is about $7 \%$, while the main bactericidal effect occurs at this frequency range.

For sterilization of products made from capillaryporous materials, high-frequency capacitive discharges (HFCD) of low pressure are most suitable, allowing for bulk treatment of materials due to volumetric nonindependent pulsed-periodic microdischarges inside the pores [32].

The effectiveness of the bactericidal effect of HFCD plasma in the air depends on the initial concentration (N0) of the test culture and the duration of treatment (t). Therefore, the survival rate of $E$. coli at N0 $\approx 1 \times 103$ $\mathrm{CFU} / \mathrm{ml}$ and $\mathrm{t}=5$ to $10 \mathrm{~min}$ is $0.2 \%$, while for $\mathrm{N} 0 \approx$ $1.4 \times 104 \mathrm{CFU} / \mathrm{ml}$ and $\mathrm{t}=7$ to $10 \mathrm{~min}$ is $71 \%$. The plasma effect on $S$. aureus is significantly lower: the survival rate of microorganisms with a 10 -minute treatment is $83 \%$ for $\mathrm{N} 0=1.8 \times 104 \mathrm{CFU} / \mathrm{ml}$ and $36 \%$ for $\mathrm{N} 0=8.0 \times$ $103 \mathrm{CFU} / \mathrm{ml}$. The sterilizing effect of plasma exposure for B. subtilis strains with $\mathrm{N} 0=1.7 \times 102 \mathrm{CFU} / \mathrm{ml}$ is also low at $\mathrm{t}=5 \mathrm{~min}$ : viable cells were isolated in an amount of $\approx 70 \%$. At $t=7$ to $10 \mathrm{~min}$, the survival of $B$. subtilis is about 2 to $3 \%$. For C. albicans with N0 $=1.0 \times 103 \mathrm{CFU} /$ $\mathrm{ml}$, a complete sterilizing effect was observed for all the durations of exposure to HFCD plasma.

R.V. Yakushin [8] studied the effect of barrier and spark discharges (amplitude 2.5 to $6 \mathrm{kV}$; frequency $45 \mathrm{kHz}$; flow rate $0.6 \mathrm{~m} 3 / \mathrm{h}$ ) on aqueous solution models containing E. coli with a concentration of $7 \times 106 \mathrm{CFU} / \mathrm{ml}$. It was shown that the barrier discharge under the given experimental conditions was ineffective against gram-negative bacteria. High inactivation of $E$. coli was noted after exposure of solution to a spark discharge. After the first two treatment cycles, a decrease in the concentration of bacteria by more than $95 \%$ compared to initial level was demonstrated.

Treatment with spark discharge allows to almost completely inactivate microorganisms in water. The experiment showed the effectiveness of spark discharge for disinfecting water at extremely high concentrations of E. coli strain. There was a decrease in the concentration of microorganisms by seven orders over 10 treatment cycles.

It may be assumed that microbial inactivation is due to oxidative destruction and integrity violation of cell membranes under the influence of the chemically active oxidant particles formed. The oxidizing agents diffusing from the water-air interface into the treated solution penetrate into microorganism cells and react with the vital macromolecules involved in metabolic processes. It is suggested that this is accompanied mainly by the oxidation of amino acids and proteins, depolarization of nucleic acids, and decomposition of other biologically active substances [8].

One of the possible factors of microbial death under the action of spark and barrier discharges is damage to the cell and its structures due to thermal or mechanical effects. Shock waves are able to intensify chemical polymerization processes and breaking of chemical bonds in the cell, thereby destroying its membrane. Moreover, there is a rupture of the bacterial cell itself [8].

Since 1990, there has been a growing interest in the use of plasma jets to inactivate bacteria, since they are best suited for practical use because of their small size and easy plasma excitation. For microbial sterilization, the most actively used plasma jets are so-called "atmosphericpressure plasma jets".

In preliminary experiments [33], the efficiency of plasma beam for inactivating bacteria was shown. In this study, E. coli bacteria were treated with plasma pencil in two different gaseous media (helium and helium mixed with $0.75 \%$ oxygen) during different time (30 and $120 \mathrm{~s}$ ). It was established that the area of the inactivated region increases with increase in exposure time, as well as with the addition of oxygen to helium, especially with increase in exposure time.

When assessing the antibacterial effects of plasma irradiation in the Scientific and Practical Center of Hygiene (Minsk, Republic of Belarus) [34], Staphylococcus aureus ATCC 6402 and Pseudomonas aeruginosa ATCC 7884 clinical isolates and E. coli ATCC 8739, E. coli ATCC 11229, Staphylococcus aureus ATCC 6538, Pseudomonas aeruginosa ATCC 15442, Bacillus subtilis ATCC 6633, Proteus mirabilis ATCC 2593, Klebsiella pneumoniae ATCC 13883 and Candida albicans ATCC 10231 museum strains were used as the study objects. The selected strains are representatives of various groups of microorganisms differing in the structure of cell membrane and defense mechanisms.

Monocultures were treated with an air plasma jet generated by a glow discharge at atmospheric pressure and direct current, at a distance of $4 \mathrm{~cm}$ between the anode and the microorganisms for $1,5,10$, and 20 minutes. The discharge current was set to $35 \mathrm{~mA}$, the air flow was 5 $\mathrm{L} / \mathrm{min}$, the temperature of the plasma jet was continuously monitored using FLIR E4 thermal imager and did not exceed $30^{\circ} \mathrm{C}$.

It was established that the pronounced antimicrobial effect of plasma irradiation with respect to monocultures of bacteria appeared only after 5 minutes of exposure. Ps. aeruginosa ATCC 15442 museum strain was the most resistant, i.e. the zone of growth inhibition after 10 minutes of exposure was only $6.8 \%$. At the same time, Ps. aeruginosa 7884, Bacillus subtilis ATCC 6633 and Kl. pneumoniae ATCC 13883 strains showed the lowest resistance, i.e. after 10 min of plasma treatment decontamination of bacteria occurred in $35.4 \%, 45.9$ and $36.0 \%$ of the plate area, respectively [34].

The bactericidal properties of the negative-corona 
low-temperature argon plasma were studied for effects on the vegetative form of E. coli strains. E.coli cultures were grown on enriched agar medium in the form of a lawn. Experimental coupons with bacteria were exposed to argon plasma, with an exposure time of 30 seconds to 5 minutes [35]. The data obtained show that the treatment of the plates with plasma jets of a low-current spark for 30 seconds leads to the death of almost all microorganisms within a radius of $1.5 \mathrm{~cm}$. The determination of the inactivation ability of argon plasma by the method of counting colonies shows that after one-minute treatment, only single colonies remain. However, the inactivation zone is not limited to the diameter of the generator nozzle, within which plasma jets of a low-current spark are formed.

Using the above data, the changes in the CFU number during the treatment of packaged dietary supplements with the low-temperature plasma were investigated. The results showed a decrease in CFU by three orders.

Research results allow to conclude that food treatment with low-temperature plasma may be one of the effective ways to reduce the growth of microorganisms, thereby increasing the shelf life and suitability without the need to maintain low-temperature conditions.

\section{The use of plasma technology in the production of meat products and to ensure their shelf life}

Recently, the plasma treatment method was tested in meat and meat products including pork [36], ham $[37,38,39,40]$, ground meat [41], sausages [42], etc. Studies have shown that atmospheric-pressure cold plasma treatment on the surface of meat products leads to the inactivation of food pathogens improving the safety and stability of meat products while increasing their shelf life. It is also effective to prevent the use of nitrite in their production.

Oehmigen et al. [43] found that plasma does not interact directly with microorganisms in a liquid, but the plasma-liquid interaction leads to liquid acidification in combination with the formation of reactive oxygen species and reactive nitrogen species including nitrate $\left(\mathrm{NO}_{3}^{-}\right)$and nitrite $\left(\mathrm{NO}_{2}^{-}\right)$, which inactivate microorganisms.

Based on this study, it was suggested that water with nitrites is formed during plasma treatment and may be used as a source of nitrites in meat products. Therefore, the aim of the study was to compare the quality of the emulsion sausages made with plasma-treated water (PTW), celery powder containing nitrite, and synthetic sodium nitrite at a concentration of $70 \mathrm{mg} / \mathrm{kg}$ and to evaluate the suitability of PTW as a source of nitrite in the production of meat products [42].

The results of the study did not show a noticeable effect on the change in color characteristics and peroxide value of sausages with PTW during storage for 28 days at $4{ }^{\circ} \mathrm{C}$ compared to samples with celery powder and sodium nitrite. Throughout the storage period, sausages with the addition of PTW had a lower concentration of residual nitrite compared to samples made with the addition of celery powder and sodium nitrite. The sensory properties of sausages made with PTW and sodium nitrite did not differ, while the sausages with the addition of celery powder received the lowest scores for flavor and consumer acceptability.

It was established [42] that the total number of aerobic bacteria was below the detection limit of $101 \mathrm{CFU} / \mathrm{g}$ for all samples on the day of manufacture (0 days) and remained at this level for the next 14 days of storage. The total number of aerobic bacteria increases to $2 \mathrm{CFU} / \mathrm{g}$ after 21 days of storage. The addition of nitrite and PTW limited the growth of aerobic bacteria on days 21 and 28 compared to the control sample. On the 28th day of storage, the total number of aerobic bacteria in the samples with plasmatreated water was similar to sausages made with nitrite solution and lower than in sausages with celery powder, although the difference was small. Color characteristics, peroxide value and sensory properties of the samples produced with PTW were similar to those of sausages with sodium nitrite. From the results obtained, it was concluded that PTW might be used as a source of nitrite.

The aim of the work conducted by the scientists of Animal Industry and Biotechnology Department, Chunnam National University, South Korea and Agricultural Biotechnology Department, Center for Food and Bioconvergence was to study the possibility of using water after plasma treatment as a source of nitrite in curing of meat products [41]. After plasma treatment of distilled water for 60 minutes, it contained $50 \mathrm{ppm}$ of nitrite. Consequently, the amount of nitrite added in ground meat by means of PTW was $0.001 \%$, while in ground meat with sodium nitrite this value reached $0.007 \%$. In order to assess the effect of PTW on the formation of meat color, various samples of ground meat were made (control ground meat without added nitrite; PTW sample ground meat with PTW; SN sample - ground meat with added sodium nitrite). After heat treatment, there was no significant difference in the values of lightness $\left(L^{*}\right)$ and yellowness $\left(b^{*}\right)$ between treatment groups. Redness $\left(a^{*}\right)$ of the heat-treated ground meat with PTW were significantly higher compared to the control group. However, $\mathrm{a}^{*}$ values of heat-treated ground meat with PTW were lower than those in samples with sodium nitrite $(\mathrm{P}<0.05)$. Thus, it can be concluded that PTW may be used as a source of nitrite in curing of meat products. However, plasma treatment system needs to be improved in order to form a large amount of nitrite in water. In addition, an appropriate classification of PTW is necessary, since PTW is neither a synthetic nor a natural source of nitrite.

Further to these studies, ham was produced with the replacement of nitrite or nitrate by plasma-treated water (PTW). To obtain PTW, distilled water was treated with plasma, after which it was injected into pork tenderloin. Ham cured with PTW (HCP sample) showed a lower 
content of aerobic bacteria ( $\log \mathrm{CFU} / \mathrm{g})$ and residual nitrite concentration, as well as a higher $\mathrm{a}^{*}$ value compared to ham cured with sodium nitrite solution (HCN sample). There were no significant differences in sensory properties between samples of HCP and HCN [40].

At week 0, HCP samples had a lower total number of aerobic bacteria compared to HCN samples $(\mathrm{p}<0.05)$. By the end of the second week of storage, the total number of aerobic bacteria in cured ham was $6.52 \mathrm{log} \mathrm{CFU} / \mathrm{g}$ for HCP samples and $6.68 \mathrm{log}$ CFU/g for HCP samples, respectively. The results are consistent with the assumptions [44] that the treatment of distilled water with a surface dielectric barrier discharge in atmospheric air has a bactericidal effect on the liquid itself.

The residual content of nitrite in all groups decreased throughout the storage time. In addition, the residual concentration of nitrite in $\mathrm{HCN}$ samples during storage was higher compared to HCP samples ( $p<0.05)$. The authors noted that the residual nitrite content is important for maintaining the quality of cured meat products during storage. However, many consumers are interested in a lower level of residual nitrite due to the potential relationship of nitrite and nitrosamines with cancer.

Scores of appearance, color, odor, flavor, juiciness, chewiness, off-odor and overall acceptability of HCP and $\mathrm{HCN}$ samples revealed no significant differences.

Thus, a number of researchers have concluded that PTW may be used as a possible substitute for synthetic nitrite [40,41] and has an antimicrobial activity [44].

Studies have been conducted on the quality of minced ham cured using atmospheric-pressure plasma (APP) treatment [40]. The minced ham was prepared using sodium nitrite, celery powder, and treated with atmospheric-pressure plasma for 30 and 60 minutes. It was established that the content of nitrite in ground meat increased with an increase in duration of treatment with PAD. The content of nitrite in ground meat after treatment with atmospheric-pressure plasma for 30 and 60 minutes reached 40.42 and $60.50 \mathrm{mg} / \mathrm{kg}$, respectively. The pink color of cured ham was formed with all types of treatment, and no significant difference in the color of cured ham $\left(\mathrm{L}^{*}, \mathrm{a}^{*}\right.$, and $\mathrm{b}^{*}$ values) was found when using different sources of nitrite. The sensory properties (flavor, color, odor, texture, general acceptability) of minced ham cured with atmospheric-pressure plasma treatment were similar to the same characteristics of minced ham cured with powdered celery. However, the flavor and general acceptability of minced ham cured with sodium nitrite received significantly lower scores compared to those for minced ham cured with celery powder or APP treatment.

The effect of atmospheric-pressure plasma (APP) treatment on nitrite levels and physicochemical parameters of ground meat models consisting of pork, water, and sodium chloride (80:20:1) was also studied during mixing. For research, a compact atmospheric-pressure plasma treatment system was developed and placed in the upper part of the ground meat mixer. It was established that the plasma treatment gradually increased the temperature of ground meat from 0.2 to $20^{\circ} \mathrm{C}$ over 60 minutes. Since the recommended final temperature of ground meat should not exceed 10 to $13^{\circ} \mathrm{C}$, plasma treatment was carried out for 30 minutes. The level of nitrites in ground meat increased with an increase in the duration of plasma treatment, reaching $65.96 \mathrm{mg} / \mathrm{kg}$ during treatment for 30 minutes. After the plasma treatment for 30 minutes, the $\mathrm{pH}$ of ground meat slightly reduced from 6.0 to 5.92. Ground meat $\mathrm{a}^{*}$ values increased significantly with an increase in plasma treatment duration, which is associated with an increase in nitrite levels. The redness value increased from $2.13 \pm 0.57$ to $7.2 \pm 0.3[45]$.

In addition, an increase in the antioxidant and antimicrobial activity of flavonoids was reported during treatment with atmospheric-pressure plasma (APP) [46].

The purpose of the work conducted by Jung S. et al. [47] was the study of nitrite level and antimicrobial activity of Perilla frutescens extracts after their treatment with atmospheric-pressure dielectric barrier plasma (APP) for their further use in production of meat products. Freeze-dried ethanol extracts of perilla treated with APP for 60 minutes contained $3.74 \mathrm{mg} / \mathrm{g}$ nitrite. The control sample (freeze-dried ethanol extracts without APP treatment) did not contain nitrite. The minimum inhibitory concentration (MIC) of APP-treated freezedried ethanol extracts for Clostridium perfringens was $200 \mu \mathrm{g} / \mathrm{ml}$. The control sample of perilla extract did not inhibit the growth of $C$. perfringens at MIC in the range of 25 to $1000 \mu \mathrm{g} / \mathrm{ml}$. The MICs of APP-treated freeze-dried ethanol extracts and controls for Salmonella typhimurium were 25 and $50 \mu \mathrm{g} / \mathrm{ml}$, respectively. Thus, new sources of nitrite with increased antimicrobial activity for use in meat technologies may be obtained from the APP-treated natural vegetable raw materials regardless of their initial nitrite level.

Thus, treatment with atmospheric-pressure plasma may be used in curing process as an alternative to the addition of nitrite. However, currently, it is difficult to give a clear classification of PTW, since it is neither a chemical reagent nor a natural source of nitrites [42]. It was suggested that the plasma treatment is a method of water purification that can remove harmful pollutants in it. In this context, PTW may be classified as purified water, containing nitrite and possessing the properties of natural antimicrobial agents.

P. Benecke et al. conducted an experiment, the purpose of which was to study the antimicrobial effect of cold atmospheric-pressure plasma on the inactivation of microorganisms on mortadella sausage slices during 21 days of storage, using S. enterica serovar Typhimurium, E. coli and L. monocytogenes as indicator microorganisms. The cold atmospheric-pressure plasma used in this study was generated by FlatPlaSter 2.0 (Terraplasma $\mathrm{GmbH}$, Garching, Munich, Germany $[\mathrm{U}=18 \mathrm{kV}, \mathrm{f}=12.5 \mathrm{kHz}, \mathrm{P}=$ $0.5 \mathrm{~V} / \mathrm{cm} 2])$ [48]. 
Low operating temperatures combined with a short plasma treatment duration $(0,30,60$, and $120 \mathrm{~s})$ at atmospheric pressure showed different effects on the inactivation of Salmonella enterica serovar Typhimurium (S.T.), Escherichia coli (E.c.) and Listeria monocytogenes (L.m.).

The effectiveness of cold plasma treatment in relation to the inactivation of microorganisms on mortadella sausage samples was limited. The maximum inactivation for S. enterica serovar Typhimurium was $0.3 \log 10$, while for E. coli and L. monocytogenes it remained unchanged. In the first two weeks of storage, the researchers did not observe significant differences in microbial counts for both L. monocytogenes and E. coli. However, after 21 days of storage, significant differences were found, i.e. 0.33 $\log 10$ (L. monocytogenes) and $0.63 \log 10$ (E. coli) when treating samples with cold atmospheric-pressure plasma for 30 and 120 s. S. enterica serovar Typhimurium were more susceptible to treatment with cold atmosphericpressure plasma compared to other studied bacteria. During storage, sausage samples treated with cold atmospheric-pressure plasma showed lower counts of S. enterica serovar Typhimurium compared to untreated control samples during the entire storage period. However, the authors noted that the differences in S. enterica serovar Typhimurium counts were quite small. This study confirms that $L$. monocytogenes are gram-positive bacteria and have thicker outer membrane compared to gram-negative $S$. enterica serovar Typhimurium and E. coli. Thus, they were less sensitive to treatment with cold atmospheric-pressure plasma. In addition, it was noted that L. monocytogenes as psychrophilic bacteria have the advantage of reproduction in a wide temperature range and may possibly grow continuously for 21 days of storage at $4 \pm 0.5^{\circ} \mathrm{C}$. Furthermore, a high content of fat $(20 \%)$ and protein (13\%) in mortadella sausage slices may also create a barrier against treatment with cold atmosphericpressure plasma [48].

One of the criteria that may be critical for the effectiveness of cold atmospheric-pressure plasma treatment is the gas used [10]. Some authors [38] consider the $\mathrm{N} 2+\mathrm{O} 2$ mixture to be the most effective for reducing the amount of bacteria on meat surface compared to using only ambient air.

Wang et al. [49] noted that cold plasma treatment of chicken fillets in a modified atmosphere $(\mathrm{O} 2 / \mathrm{CO} 2 / \mathrm{N} 2=$ $65 / 30 / 5)$ with an increased oxygen concentration allowed reducing the bacterial load by at least two log CFU/g during 14 days of storage.

Chinese scientists conducted studies concerning the effect of dielectric barrier discharge (DBD) plasma on microbial inactivation and discoloration of the pork loin surface [50].

Treatment duration with dielectric barrier discharge (DBD) plasma of pork loin inside sealed polypropylene trays was 60,120 , and $180 \mathrm{~s}$ at $80 \mathrm{kV}$ and room temperature. After plasma treatment, samples were stored at room temperature for 24 hours.

After treatment of pork loin with DBD using gaseous media, i.e. air, $40 \% \mathrm{O} 2$-MAP and $60 \%$ O2-MAP, the survival rate of aerobic bacteria decreased by $15.2 \%, 26.7 \%$, and $32.4 \%$ when exposed for $60 \mathrm{~s}$ and $26.2 \%, 39.4 \%$, and $45.1 \%$ when exposed for $120 \mathrm{~s}$, respectively. With an increase in treatment duration up to $180 \mathrm{~s}$, the survival rate of aerobic bacteria decreased by $42.5 \%, 49 \%$, and $53.2 \%$ when using gaseous media, i.e. air, $40 \%$ O2-MAP and $60 \% \mathrm{O} 2-\mathrm{MAP}$, respectively.

There were no significant differences in color characteristics between plasma-treated samples regardless of the treatment duration. So, with a higher oxygen concentration of $60 \%$ at treatment duration of $120 \mathrm{~s}$, L $\mathrm{L}^{*}$ values in pork loin decreased from 51.2 to 50.23 . It was determined that $\mathrm{a}^{*}$ valueis sensitive to oxygen concentration and plasma treatment duration. This is consistent with the findings by Attri et al. [51], who determined that the structure of hemoglobin changed in the presence of particles with high reactivity generated using DBD plasma in the presence of various gases. The authors suggested that DBD plasma also induces a change in the structure of myohemoglobin, which may affect $\mathrm{a}^{*}$ values in meat. As for $b^{\star}$ values, they significantly increased with increasing oxygen concentration and treatment duration. Similarly, $\mathrm{b}^{*}$ values in bacon did not change when helium was used, but increased at higher input power when helium/oxygen was used [16].

The effectiveness of plasma treatment depends on the surface of the treated medium. Joo-Sung Kim et al. [52] studied the effect of atmospheric-pressure plasma using argon on the inactivation of $S$. aureus placed on polystyrene plates, in agar plates and inoculated on the surface of dried beef cut into $5 \times 5 \mathrm{~cm}$ pieces, depending on the duration of treatment. It was established that after 2 minutes of treatment, the amount of $S$. aureus ATCC 12600 reduced by 3-4 log CFU/g on polystyrene and agar, but on a sample of dried beef, this effect was achieved only after treatment for 10 minutes. This allowed the authors to suggest that surface properties may significantly affect the degree of $S$. aureus inactivation by plasma. No significant changes in fatty acid composition, color and shear force for dried beef samples were found.

Analysis of $S$. aureus morphological changes on the surface of dried beef after the plasma treatment was carried out by scanning electron microscopy and showed that within 5 minutes after treatment, Staphylococcus aureus cells were severely damaged with the formation of many holes in membrane. Optical spectrum analysis suggested that reactive oxygen species, especially the singlet oxygen at $777 \mathrm{~nm}$, are mainly responsible for the inactivation and cellular deformation of $S$. aureus.

Bacteria on irregular surface migrate to a depth of approximately 140 microns due to the capillary effect. At these distances from the surface, microorganisms in general are not exposed to the sterilizing treatment. 
In addition, ruptures resulting from severe contraction of muscle fibers may provide pathways for bacteria penetration into a depth. All these factors affect the efficiency of sterilization by the dielectric barrier discharge plasma.

Preliminary results of the studies to justify the possibility of using cold argon plasma for sterilizing canned meat and fish products and to evaluate the sterilizing effect of cold argon plasma that were carried out using one-day cultures of Ps. fluorescens, St. aureus, and E. coli with an initial concentration of $5 \times 104 \mathrm{CFU} / \mathrm{ml}$ in $50 \mathrm{ml}$ of normal saline [53] showed that plasma treatment for 10 minutes results in almost complete elimination of vegetative cells and spores. Furthermore, and it was established that with plasma-based sterilization, vitamins in canned model vegetable/fish mixtures are better preserved.

The results of the study showed the sterilizing ability of low-temperature plasma for different types of microorganisms and the possibility of its using as an effective method for treatment of meat products to increase their shelf life.

\section{Conclusion}

Currently, plasma technologies, despite their great potential, are not available as a sterilizing tool in food industry, mainly due to the lack of studies for the plasma discharge effect on food chemical components, interactions with packaging materials, etc., as well as applicability in industrial production. The use of cold non-thermal plasma in food technology still needs to be thoroughly studied, despite the data obtained on the effectiveness of this type of treatment in the inactivation of food pathogens or natural toxins and, as a result, to increase the shelf life of food products.

\section{REFERENCES}

1. Advanced cold plasma technology. NPC "Plasma", 2016. [Electronic resource: http://plasmamed.ru/application/ files/8114/8828/3627/RUS-PRESENTATION-NPC_Plasma_ investors.pdf . Access date: 09.10.2018]. (In Russian)

2. Tikhonov, E.A. (2013). Investigation of the influence of cold plasma treated water on potato planting growth intensity and productivity. Polythematic online scientific journal of Kuban State Agrarian University, 85, 363-373. (In Russian)

3. Ur'eva, A. V., Kovalchuk, A.N. (2014). Introduction to plasma technology and hydrogen energy: a tutorial. Tomsk: Tomsk Polytechnic University. - 90 p. (In Russian)

4. Fridman, G., Friedman, G., Gutsol, A., Shekhter, A.B, Vasilets, V.N., Fridman, A. (2008). Applied Plasma Medicine. Plasma Processes and Polymers, 5(6), 503-533. DOI: 10.1002/ ppap.200700154

5. Avdeev, S.M., Kuznetsova, E.A., Sosnin, E.A. Plasma treatment of atmospheric pressure of contaminated Escherichia coli surfaces. [Electronic resource: http://asf.ural.ru/VNKSF/Uchastniki/ vnksf11/tezis/04/AvdeevSM/AvdeevSM.html. Access date: 09.10.2018]. (In Russian)

6. Smirnov, B.M. (1982). Introduction to plasma physics. M.: Nauka. - 176 p. (In Russian)

7. Yang, L., Chen, J., Gao, J. (2009). Low temperature argon plasma sterilization effect on pseudomonas aeruginosa and its mechanisms. Journal of Electrostatics, 67(4), 646-651. DOI: 10.1016/j.elstat.2009.01.060

8. Yakushin, R.V. (2015). Intensification of the redox potential of processes in aqueous solutions using the electric discharge plasma method. Dissertation for the scientific degree of Candidate of Technical Sciences. M.: RUCT named after D.I. Mendeleev. -163 p. (In Russian)

9. Baldanov, B.B. (2017). Sources of non-equilibrium argon plasma based on low-current high-voltage discharges. Dissertation for the scientific degree of Doctor of Technical Sciences. Tomsk: Tomsk State University of Control Systems and Radioelectronics. -239 p. (In Russian)

10. Misra, N.N., Jo, C. (2017). Applications of cold plasma technology for microbiological safety in meat industry. Trends in Food Science \& Technology, 64, 74-86. DOI: 10.1016/j. tifs.2017.04.005

11. Moisan, M., Barbeau, J., Crevier, M.-C., Pelletier, J., Philip, N., Saoudi, B. (2002). Plasma sterilization. Methods and mechanisms. Pure and Applied Chemistry, 74(3), 349-358. D0I: 10.1351/ pac200274030349

12. Moisan, M., Barbeau, J., Moreau, S., Pelletier, J., Tabrizian, M., Yahia, L'H. (2001). Low-temperature sterilization using gas plasmas: a review of the experiments and an analysis of the inactivation mechanisms. International Journal of Pharmaceutics, 226(1-2), 1-21. DOI: 10.1016/S0378-5173(01)00752-9

13. Rahul, R., Stan, O., Rahman, A., Littlefield, E., Hoshimiya, K., Yalin, A.P., Sharma, A., Pruden, A., Moore, C.A., Yu, Z., Collins, G.J. (2005). Optical and RF electrical characteristics of atmospheric pressure open-air hollow slot microplasmas and application to bacterial inactivation. Journal of Physics D: Applied Physics, 38(11), 1750-1759. DOI:10.1088/0022-3727/38/11/016 14. Opretzka, J., Benedikt, J., Awakowicz, P., Wunderlich, J., von Keudell, A. (2007). The role of chemical sputtering during plasma sterilization of Bacillus atrophaeus. Journal of Physics D: Applied Physics, 40(9), 2826-2830. DOI:10.1088/0022-3727/40/9/024 15. Ivanova, I.P., Trofimova, S.V., Piskarev, I.M., Knyazev, D.I., Timush, A.V., Burkhina, O.E., Litvinova, L.G. (2011). The effect of active forms of oxygen of low-temperature gas-discharge plasma on the resistance of cell membranes. Vestnik of Lobachevsky University of Nizhni Novgorod, 2-2, 190-195. (In Russian)

16. Christofi, N., Anpilov, A.M., Barkhudarov, E.M., Kop'ev, V.A., Kossyi, I.A., Taktakishvili, M.I., Zadiraka, Y. (2002). Pulsed high voltage electric discharge disinfection of microbially contaminated liquids. Letters in applied microbiology, 35(1), 90-94. DOI: 10.1046/j.1472-765X.2002.01139.x

17. Laroussi, M., Mendis, D.A., Rosenberg, M. (2003). Plasma interaction with microbes. New Journal of Physics, 5(4), 41.141.10. DOI: $10.1088 / 1367-2630 / 5 / 1 / 341$

18. Mendis, D.A., Rosenberg, M., Azam, F. (2000). A note of possible electrostatic disruption of bacteria. IEEE Transactions on Plasma Science, 28(4), 1304-1306. D0I: 10.1109/27.893321

19. Boudam, M.K., Moisan, M., Saoudi, B., Popovici, C. Gherardi, N., Massines, F. (2006). Bacterial spore inactivation by atmospheric pressure plasmas in the presence or absence of UV photons as obtained with the same gas mixture. Journal of Physics D: Applied Physics, 39(16), S07, 3494-3507. D0I: 10.1088/00223727/39/16/S07

20. Weltmann, K.-D., von Woedtke Th. (2011). Basic requirements for plasma sources in medicine. EPJ Applied Physics, 55(1), ap100452. DOI: 10.1051/epjap/2011100452

21. Kobzev E.N., Kireev G.V., Rakitskii Y.A., Martovetskaya I.I., Chugunov V.A., Kholodenko V.P., Khramov M.V., Akishev Y.S., Trushkin N.I., Grushin M.E. (2013). Effect of cold plasma on the E. coli cell wall and plasma membrane. Applied Biochemistry and Microbiology, 49, 2, 144-149.

22. Becker, K., Koutsospyros, A., Yin, S.M., Christodoulatos, C., Abramzon, N., Joaquin, J.C., Brelles-Marĩo, G.(2005). Environmental and biological applications of microplasmas. Plasma Physics and Controlled Fusion, 47(12B), B513-B523. DOI: 10.1088/07413335/47/12B/S37

23. Laroussi, M., Leipold, F. (2004). Evaluation of the roles of reactive species, heat, and UV radiation in the inactivation of bacterial cells by air plasmas at atmospheric pressure. International Journal of Mass Spectrometry, 233(1-3), 81-86. DOI: 10.1016/j. ijms.2003.11.016

24. Siemens, W. Uber die elecktrostatische induction und die verzogerung des stroms in flaschendraten. Poggendorfs Ann. Phys. Chem. - 1857. - V. 12, 66-122.

25. Abou-Ghazala, A., Katsuki, S., Schoenbach, K.H., Dobbs, F.C. Moreira, K.R. (2002). Bacterial decontamination of water by 
means of pulsed corona discharges. IEEE Transactions on Plasma Science, 30(4), 1449-1453. DOI: 10.1109/TPS.2002.804193

26. Laroussi, M. (1996). Sterilization of contaminated matte with atmospheric pressure plasma. IEEE Transactions on Plasma Science, 24(3), 1188-1191. DOI: 10.1109/27.533129

27. Birmingham, J.G., Hammerstrom, D.J. (2000). Bacterial decontamination using ambient pressure nonthermal discharges. IEEE Transactions on Plasma Science, 28(1), 51-55. DOI: 10.1109/27.842862

28. Kayes, M.M., Golden, D.A., Hulbert, G., Roth, J.R., Morrison, J., Montie, T.C., Kelly-Wintenberg, K. (2000). Killing of pathogenic food-borne bacteria exposed to a one atmosphere uniform glow discharge plasma(OAUGDP). Proceedings of 27th IEEE International conference of plasma sciences (Cat. No.00CH37087), P. 251.

29. Sun, Y., Qiu, Y., Nie, A., Wang, X. (2007). Experimental research on inactivation of bacteria using dielectric barrier discharge. IEEE Transactions on Plasma Science, 35(5), 1496-1500. DOI: 10.1109/ TPS.2007.905947

30. Bray, B.J.J., Brelles-Marino, J.C., Abramzon, N. (1999) Destruction of bacterial communities using gas discharge plasma. Proceedings of 26th IEEE International conference of plasma sciences (Cat. No.99CH36297), P. 154

31. Vleugels, M., Shama, G., Deng, X.T., Greenacre, E., Brocklehurst, T., Kong, M.G. (2005). Atmospheric plasma inactivation of biofilm forming bacteria for food safety control. IEEE Transactions on Plasma Science, 33(2), 824-828.

32. Azharonok, V.V., Kratko, L.E, Filatova, I.I., Melnikova, L.A. Dudchik N.V., Yanetskaya S.A. (2008). Inactivation of microorganisms in the plasma of high-frequency capacitive and low-pressure barrier discharges. V International Symposium on Theoretical and Applied Plasma Chemistry, 2, 414-417. (In Russian) 33. Laroussi, M., Tendero, C., Lu, X., Alla, S., Hynes, W.L. (2006). Inactivation of bacteria by the plasma pencil. Plasma process and Polimers, 3(6-7), 470-473. DOI: 10.1002/ppap.200600005

34. Dudchik, N.V., Emeliyanova, O.A., Kazak, A.V., Kirillov, A.A., Simonchik, L.V. (2017). Estimation of biological effect of air plasma jet in model experiment. Health and environment, 27, 20-23. (In Russian)

35. Gomboeva, S.V., Badmaeva, I.I., Baldanov, B.B., Ranzhurov, Ts.V. (2016). Use of low temperature plasma in the food industry. Materials of the I International Scientific and Technical Conference (extramural), 69-72. (In Russian)

36. Fröhling, A., Durek, J., Schnabel, U., Ehlbeck, J., Bolling, J., Schlüter, 0. (2012). Indirect plasma treatment of fresh pork Decontamination efficiency and effects on quality attributes. Innovative Food Science and Emerging Technologies, 16, 381-390. DOI: 10.1016/j.ifset.2012.09.001

37. Lee, H.J., Jung, H., Choe, W., Ham, J.S., Lee, J.H., Jo, C. (2011). Inactivation of Listeria monocytogenes on agar and processed meat surfaces by atmospheric pressure plasma jets. Food microbiology, 28(8), 1468-1471. DOI: 10.1016/j.fm.2011.08.002

38. Song, H.P., Kim, B., Choe, J.H., Jung, S., Moon, S. Y., Choe, W., Jo, C. (2009). Evaluation of atmospheric pressure plasma to improve the safety of sliced cheese and ham inoculated by 3-strain cocktail Listeria monocytogenes. Food Microbiology, 26(4), 432436. DOI: 10.1016/j.fm.2009.02.010

39. Lee, J., Jo, K., Lim, Y., C. Jo, Choe, J., Jung, S. (2017). Quality properties of ground ham cured by atmospheric pressure plasma treatment. Proceedings of the 63th International Congress of Meat Science and Technology, Cork, Ireland, 574.

40. Hae, I.Y., Hyun-Joo, K., Jun, H.Ch., Hee-Jun, J., Samooel, J., Sanghoo, P., Wonho, C., Cheorun, J. (2015). The use of plazma- treated water as source of nitrite for curing ham. Proceedings of the 61th International Congress of Meat Science and Technology, France, 7.26

41. Samooel, J., Hyun, J. K., Sanghoo, P., Hae In. Y., Wonho, C., Cheorun, Jo (2015). The addition of nitrite to processed meat by plazma-treated water. Proceedings of the 61th International Congress of Meat Science and Technology, France, 7.25.

42. Jung, S., Kim, H.J., Park, S., In Yong, H., Choe, W., Jo, C. (2015) The use of atmospheric pressure plasma-treated water as a source of nitrite for emulsion-type sausage. Meat Science, 108, 132-137. DOI: 10.1016/j.meatsci.2015.06.009

43. Oehmigen, K., Hahnel, M., Brandenburg, R., Wilke, C. Weltmann, K.D., von Woedtke, T. (2010). The role of acidification for antimicrobial activity of atmospheric pressure plasma in liquids. Plasma Processes and Polymers, 7(3-4), 250-257. D0I: 10.1002/ ppap.200900077

44. Oehmigen, K., Winter, J., Hahnel, M., Wilke, C., Brandenburg, R., Weltmann, K. D., von Woedtke, T. (2011). Estimation of possible mechanisms of Escherichia coli inactivation by plasma treated sodium chloride solution. Plasma Processes and Polymers, 8(10), 904-913. DOl: 10.1002/ppap.201000099

45. Jung, S., Lee, J., Lim, Y., Choe, W., Yong, H.I., Jo, C. (2017). Direct infusion of nitrite into meat batter by atmospheric pressure plasma treatment. Innovative Food Science and Emerging Technologies, 39, 113-118. DOI: 10.1016/j.ifset.2016.11.010

46. Kim H.J., Yong H.I., Park S., Kim K., Kim T.H., Choe W., Jo C. (2014). Effect of atmospheric pressure dielectric barrier discharge plasma on the biological activity of naringin. Food Chemistry, 160, 241-245. DOI: 10.1016/j.foodchem.2014.03.101

47. Jung, S., Jo, K., Lee, J., Yong, H.I., Yum, S.J., Jeong, H.G., Jo, C. (2017). Development of natural nitrite source by atmospheric pressure plasma. Proceedings of the 63th International Congress of Meat Science and Technology, Cork, Ireland, 562.

48. Benecke, P., Ahlfeld, B., Boulaaba, A., Zimmermann, J.L., Klein, G. (2016). Effect of atmospheric cold plasma (ACP) on Escherichia coli, Listeria monocytogenes and Salmonella enterica serovar Typhimurium on ready-to-eat mortadella-type sausage. Proceedings of the 62th International Congress of Meat Science and Technology, Bangkok, Thailand, № 06-09.

49. Wang, J., Zhuang, H., Hinton, A., Zhang, J. (2016). Influence of in-package cold plasma treatment on microbiological shelf life and appearance of fresh chicken breast fillets. Food Microbiology, 60 142-146. DOI: 10.1016/j.fm.2016.07.007

50. Mingming, Huang, Jiamei Wang, Wenjing Yan, Weiwei Qiao, Jianhao Zhang (2016). Effect of dielectric barrier plasma on bacteria and surface color of pork loin. Proceedings of the 62th International Congress of Meat Science and Technology, Bangkok, Thailand, № P 09-25.

51. Attri, P., Sarinont, T., Kim, M., Amano, T., Koga, K., Cho, A. E., Choi, E. H., Shiratani M. (2015). Influence of ionic liquid and ionic salt on protein against the reactive species generated using dielectric barrier discharge plasma. Scientific Reports, 5, 17781 DOI: 10.1038/srep17781

52. Kim, J.-S., Lee, E.-J., Choi, E.H., Kim, Y.-J. (2014). Inactivation of Staphylococcus aureus on the beef jerky by radio-frequency atmospheric pressure plasma discharge treatment. Innovative Food Science and Emerging Technologies, 22, 124-130. DOI: 10.1016/j.ifset.2013.12.012

53. Kasyanov, D.G., Zaporogskiy, A.A. (2013). The use of cold argon plasma to sterilize canned meat and fish products. Collection of materials of the International Scientific and Technical Internet Conference, Krasnodar, 12-14. (In Russian)

\section{AUTHOR INFORMATION}

Nataliya A. Gorbunova - candidate of technical sciences, Scientific secretary, V.M. Gorbatov Federal Research Center for Food Systems of Russian Academy of Sciences, 109316, Moscow, Talalikhina str., 26 . Tel.: +7 -495-676-93-17. E-mail: n.gorbunova@fncps.ru

Contribution

The author completely prepared the manuscript and is responsible for plagiarism

Conflict of interest

The author declare no conflict of interest 